\title{
Endovascular management of basilar artery occlusion secondary to fusiform aneurysm with intraluminal thrombus
}

\author{
André Beer-Furlan, MD, Hormuzdiyar H. Dasenbrock, MD, Krishna C. Joshi, MBBS, MCh, \\ and Michael Chen, MD
}

Department of Neurological Surgery, Rush University Medical Center, Chicago, Illinois

Acute basilar artery occlusion is one of the most devastating subtypes of ischemic stroke with an extremely high morbidity and mortality rate. The most common causes include embolism, large-artery atherosclerosis, penetrating small-artery disease, and arterial dissection. The heart and vertebral arteries are the main source of emboli in embolic basilar occlusions. The authors present an uncommon acute basilar occlusion secondary to a fusiform aneurysm with intraluminal thrombus. The patient underwent a mechanical thrombectomy with successful recanalization, but persistent intraluminal thrombus. The authors discuss the management dilemma and describe their choice for placement of flow diverter stents.

The video can be found here: https://youtu.be/XzBdgxJPSWQ.

KEYWORDS endovascular; basilar artery occlusion; fusiform aneurysm; mechanical thrombectomy; flow diverter stent; video 